\title{
La certificación de las competencias comunicativas del español para inmigrantes
}

\author{
Marta Baralo Ottonello \\ Susana Martín Leralta \\ Universidad Antonio de Nebrija \\ IsABEL PASCUAL GómeZ \\ Universidad de Alcalá
}

Recibido: 8 enero 2015 / Aceptado: 5 mayo 2015

ISSN: $1697-7467$

\begin{abstract}
RESUMEN: Este trabajo muestra un proceso de certificación de las competencias comunicativas del español para inmigrantes. El proyecto ${ }^{1}$ pasó por las siguientes fases: análisis de las necesidades comunicativas de la población inmigrante, diseño del diploma, formación, difusión pública y evaluación. Colaboraron en este proceso 295 candidatos y 30 instituciones dedicadas a la ayuda al inmigrante. La obtención del diploma (97,1\% de los presentados) constituye el logro de una meta personal porque incrementa sus habilidades comunicativas, favorece su plena integración y favorece el proceso de normalización en la comunidad de acogida.

Palabras clave: competencia comunicativa, certificación, formación de profesores de español como segunda lengua, integración cultural.
\end{abstract}

\section{Certification of Communicative Competences in Spanish for Immigrants}

\begin{abstract}
This work shows a project of acquisition of the communicative linguistic competences of the immigrant population and its official certification. It was developed in the following phases: by analysing the immigrant population's communicative needs, designing the diploma, making the public aware, training examiners and assessing the project. 295 participants and 30 centres undergoing the language certificate process. For many immigrants, obtaining this certificate $(97.1 \%)$ is a personal achievement because it increases their basic communication competences, which favours their full integration into the society, and thus, helping them adapt better to their welcoming community.

Keywords: communicative competence, certification, teacher training on Spanish as a second language, cultural integration.
\end{abstract}

\section{INTRODUCCIÓN}

El trabajo que se presenta tiene como objetivo mostrar la implantación de un proceso de evaluación y certificación de la competencia lingüística del español para la población inmigrante de Madrid. Surge con la intención de ofrecer una mejor respuesta de carácter socioeducativo a las necesidades comunicativas reales de esta población.

\footnotetext{
${ }^{1}$ I+D INMIGRA2007 (referencia S2007/HUM 0475) financiado por la Comunidad de Madrid.
} 
Aunque ya existían certificados de español para extranjeros en algunas instituciones españolas e hispanoamericanas, el proceso de estandarización de la certificación lingüística en español se inicia en 2004, en el seno del III Congreso Internacional de la Lengua Española celebrado en Argentina. Este se materializa en Colombia en 2007 en el acuerdo de creación del Sistema Internacional de Certificación del español como lengua extranjera (SICELE) cuya misión es armonizar los criterios de gestión de los sistemas de certificación del conocimiento del español a hablantes de otras lenguas, promover el uso de buenas prácticas y facilitar el reconocimiento oficial de los diplomas.

Sus requisitos están recogidos en los estándares de calidad de tres agencias internacionales: The Association of Language Testers of Europe (ALTE), European Association for Language Testing and Assessment (EALTA), e International Language Testing Association (ILTA). Sus directrices para la buena práctica en el desarrollo de pruebas y la evaluación de lenguas son un estándar y se han tenido en consideración para la implantación de la prueba de evaluación que se presenta. Según LLorián (2012) la certificación del español, promovida por organizaciones de países de habla hispana, se encuentra prácticamente concentrada en unos cuantos grandes sistemas de exámenes. Nos referiremos únicamente a los sistemas que dependen de instituciones certificadoras pertenecientes a países hispanohablantes y que reúnen las siguientes características: se administran en áreas geográficas de gran extensión y de alcance internacional; cuentan con grupos numerosos de candidatos; integran exámenes de alto perfil (tienen importantes consecuencias en la vida de los candidatos). Consideramos dentro de este grupo los siguientes: DELE (Diploma de Español como Lengua Extranjera), del Ministerio de Educación del Gobierno de España; CELU (Certificado de Español Lengua en Uso), promovido por un consorcio de universidades públicas de la República Argentina; CELA (Certificados de Español como Lengua Adicional), dependiente del Centro de Enseñanza para Extranjeros de la Universidad Nacional Autónoma de México; DIE (Diploma Internacional de Español) de la Fundación FIDESCU; DUCLE (Diploma Universitario de Competencia en Lengua Española), desarrollado y administrado por la Universidad Nacional de Rosario (Argentina); los certificados del Instituto de Formación Empresarial (IFE) de la Cámara Oficial de Comercio, Industria y Navegación de Madrid, en colaboración con la Universidad de Alcalá, y los certificados ELYTE (Evaluación Lingüística y de Técnicas Empresariales de Español de Empresa) de la Cámara Oficial de Comercio de España en Francia, que opera bajo la tutela del Ministerio de Industria del Gobierno de España. Es significativo que cuatro de estos siete sistemas aludan en sus denominaciones a fines específicos de uso de la lengua. Los cuatro últimos de esta lista incluyen exámenes que certifican la habilidad de uso del español en contextos específicos, profesionales o académicos.

La prueba de certificación que aquí se presenta tiene como referente un modelo de evaluación de la competencia comunicativa que se centra en evaluar los significados de los mensajes más que en la evaluación de aspectos formales de este (Luoma, 2004; García Mateos, 2004; Bordón, 2006; Instituto Cervantes, 2006; Villalba y Hernández, 2007; Estaire, 2009). Este modelo evalúa competencias sobre la capacidad de comprender qué es lo que se expresa en un texto oral o escrito, o la capacidad de expresar de forma oral o escrita lo que deseamos transmitir. Exige por lo tanto de la comprensión, negociación y expresión de significados con el fin de alcanzar una finalidad comunicativa, considerando que el logro de 
esta en una tarea de comunicación se pone de manifiesto si se reflejan las correspondientes adquisiciones en competencias lingüísticas, sociolingüísticas, pragmáticas y estratégicas (las microcompetencias que conforman la competencia comunicativa), tal como se detalla en Baralo y Estaire (2010). Este tipo de evaluación por competencias comporta distintos efectos socioprofesionales como la certificación, reconocimiento, convalidación de experiencia, etc. (Martínez y Echeverría, 2009). Tal como indica Luoma (2004: 162), hay tres líneas guía para evaluar la competencia comunicativa: (1) orientarla a la lengua, (2) orientarla a la comunicación y (3) basarla en una situación concreta o contexto. Este contexto se basa no solo en la cultura, sino también en aspectos como el lugar en que tiene lugar ese encuentro comunicativo, las relaciones que se dan entre los interactuantes (amistad, laboral, amorosa...) y el motivo de esa comunicación (Vilà, 2006). Estas son las líneas guía que aquí se han seguido. Por ello y dadas las necesidades lingüísticas comunicativas del grupo meta, el examen de certificación evalúa la competencia del candidato de una manera asimétrica, esto es, dando más valor a las destrezas orales que a las escritas, y a las destrezas receptivas que a las productivas, de manera que la comprensión auditiva y la interacción oral son las que más peso tienen en el total de la nota, seguidas por la comprensión lectora y la expresión escrita.

La certificación que se propone sigue el enfoque metodológico propuesto por Estaire (2009) y Luoma (2004:187), quienes ponen el foco en la evaluación de las competencias del candidato para estructurar la información de manera efectiva y comunicarse con fluidez de una manera socialmente aceptada. Este enfoque en las tareas de evaluación resulta el más adecuado si se pretende una coherencia con el enfoque empleado en la didáctica del español como lengua nueva, al tiempo que contribuye al impacto positivo del examen sobre el proceso de enseñanza/aprendizaje. La elección del enfoque metodológico para el diseño de las tareas de evaluación también se encuentra en consonancia con las últimas propuestas de enseñanza de español a inmigrantes (García Mateos, 2004; Instituto Cervantes, 2006; Villalba y Hernández, 2007; Herrero 2013), grupo meta al que se dirige el examen de certificación.

\section{Objetivos}

Mediante este proceso de evaluación y certificación, similar a la que se está ofreciendo ya en otros países, se pretende:

- Implantar un procedimiento de certificación de la competencia de la lengua española que garantice una cierta autonomía comunicativa en contextos laborales, para inmigrantes adultos que deseen aprender español.

- Contribuir a la integración de la población inmigrante adulta facilitándole la adquisición de competencias que le permitan comunicarse en los diferentes contextos de la vida social, en particular, la laboral y la administrativa.

- Contribuir a que las instituciones que atienden a los inmigrantes adultos ofrezcan programas de aprendizaje de la lengua que conduzcan de forma rápida y eficaz a la consecución de una competencia comunicativa, que les posibilite una interacción autónoma con los hablantes nativos. 


\section{Metodología}

El proceso se desarrolló en cuatro fases con proceso de evaluación continua para realizar los ajustes necesarios sobre la programación inicial: análisis de necesidades, diseño de la prueba, formación de los evaluadores y realización de la certificación.

\subsection{Análisis de necesidades}

Como fase previa al diseño de la prueba de evaluación se consideró imprescindible conocer, en primer lugar, las necesidades de las distintas comunidades implicadas en el proceso comunicativo. Por este motivo, el proyecto tuvo como punto de partida la obtención de un análisis de las necesidades comunicativas de la población inmigrante y de los profesionales que conviven en su entorno. Se entendió que como paso previo era necesario:

a) Saber más acerca de la biografía lingüística del inmigrante que llega a la Comunidad de Madrid (formación académica, estancias previas, estudios de español en el país de origen, etc.).

b) Conocer las necesidades socioculturales del inmigrante en relación con los ámbitos de uso de su comunidad discursiva.

c) Conocer el tipo de trabajo que desarrolla el inmigrante y las necesidades lingüísticas de esa profesión.

Los estudios de observación etnográfica no participante, las entrevistas personales realizadas a profesionales en contacto con la inmigración, y las encuestas a profesores con experiencia en enseñanza del español a inmigrantes ayudaron a conocer estas necesidades. Se observaron y registraron las interacciones comunicativas de inmigrantes de lengua nativa diversa al español, residentes en la Comunidad de Madrid, con diversos agentes de la administración pública y de servicios básicos, y con los contratistas más habituales o empresarios de aquellos sectores que requieren trabajadores provenientes de la inmigración. En total se realizaron 45 observaciones. Los participantes procedían de muy diversas nacionalidades $(\mathrm{Ru}-$ manía, China, Nigeria, Marruecos, Egipto, Senegal, Finlandés, Moldavia, Ucrania, Palestina, etc.) y con perfiles muy diferentes en cuanto a formación y profesión. En su mayoría, se trataba de adultos de entre 20 a 45 años. Para poder llevar a cabo esta observación, dadas las características tan peculiares de la muestra objeto del estudio, se tuvo que realizar un muestreo intencional, contando con la colaboración constante de los coordinadores de 5 de los $\mathrm{CEPI}^{2}$ participantes en el proyecto y de otras organizaciones. Estos centros han actuado como mediadores con la población inmigrante ajustando las citas entre el investigador y el inmigrante. El equipo investigador observó y registró una serie de situaciones comunicativas iniciales en los ámbitos y tareas más comunes y básicas. De los ámbitos individuales, sociales y profesionales, se seleccionaron las tareas de comunicación más comunes y necesarias para la vida cotidiana de los sujetos de estudio: salud, asesoría laboral, asesoría jurídica, transporte, actividades en bancos, centros de enseñanza del español, ayuntamientos, etc. En el registro

\footnotetext{
${ }^{2}$ Centros de Participación e Integración de Inmigrantes de la Comunidad de Madrid.
} 
y transcripción de los datos se tuvieron en consideración los intercambios lingüísticos, los aspectos contextuales, los aspectos comunicativos no verbales y otros datos relevantes. Los datos obtenidos se cruzaron con encuestas online realizadas a 35 profesores de español. Estas encuestas aportaron la valoración de los profesores que trabajan con inmigrantes sobre los niveles A1 y A2 del Plan curricular del Instituto Cervantes y del Marco común europeo para la enseñanza, aprendizaje y evaluación de las lenguas (en adelante, MCER), ajustando así el nivel A1 al nivel propuesto A2-n (considerando "n" el control gramatical), según su experiencia con los sujetos observados.

Los resultados del contraste entre los datos provenientes de estas encuestas y los provenientes del trabajo de campo permitieron clarificar y definir aquello que realmente constituye, o debe constituir, la competencia comunicativa básica de los destinatarios de la certificación lingüística, y abordar el diseño del examen (Aguado-Orea y Baralo, 2007; Escudero y Guerra, 2009; Baralo, 2009; Martín, 2009 y 2011).

\subsection{Diseño del examen}

Para establecer el diseño y determinar las pautas más idóneas para su gestión se tuvieron en cuenta los factores relativos a la administración del programa, al estudio de costes de formación y certificación por persona y a las redes de colaboración. Los referentes internacionales que avalan la propuesta de certificación son las propuestas realizadas por las agencias ALTE (Association of LanguageTesters of Europe), EALTA (European Association for LanguageTesting and Assessment) e ILTA (International Language Testing Association). Entre los documentos más relevantes tenidos en cuenta se pueden mencionar el Código de práctica de ALTE, los Estándares mínimos para el establecimiento de perfiles de calidad en los exámenes de ALTE y las Directrices de EALTA para una buena práctica en el desarrollo de pruebas y la evaluación de lenguas. Estas directrices están, además, en consonancia con las recomendaciones del MCER (Consejo de Europa, 2002).

Las etapas o logros de esta fase fueron:

a) Elaboración de las especificaciones iniciales del examen de certificación. Para la elaborar las especificaciones iniciales, se contó con la colaboración de expertos en las diversas áreas implicadas: evaluación, didáctica de L2, formación de profesores, investigación cuantitativa y estadística aplicada a la educación. Según (Alderson, Clahpham y Wall ,1998: 14) las especificaciones del examen consisten en un documento, normalmente de uso interno, que facilita "información oficial sobre lo que un examen evalúa y cómo lo evalúa". Un paso esencial en el desarrollo de exámenes de idiomas "es hacer operativos los constructos que queremos medir" (Bachman, 1990: 153-154). Este documento se elabora para intentar asegurar la equivalencia de las pruebas, esto es, que los diferentes ítems o formas del examen son equivalentes en contenido, o que el contenido del examen representa adecuadamente el contenido del programa.

b) Diseño del examen piloto. Para el diseño de las pruebas se tuvo en consideración que las tareas de comunicación que se van a evaluar deben reproducir y activar procesos auténticos de comunicación, oral o escrita, poniendo en juego estrategias 
de comunicación como las que utilizamos en la vida cotidiana, en las fases de planificación, ejecución, control y reparación de la comunicación. La prueba se sitúa en un nivel A1+1 o en un nivel A2-n del MCER. Para la elaboración de su contenido se siguieron sus descriptores adaptados a la población meta. La certificación consta de cuatro pruebas, correspondientes a cada una de las destrezas lingüísticas comunicativas: comprensión lectora, comprensión audiovisual, expresión e interacción escrita, y expresión e interacción oral. La especificación y distribución de tareas, así como los tiempos asignados son los que siguen:

- Prueba de comprensión lectora: consta de 4 tareas y una duración de 20 minutos. El candidato debe ser capaz de leer diversas informaciones y relacionarlas con un tema, con el tipo de información ofrecida, y con el lugar en el que puede aparecer o contexto en el que puedan ser de interés. Se han incluido diferentes capacidades de comprensión de diferentes géneros textuales, entre las que se destacan la posibilidad de comprender en español la idea principal de un cartel u hoja de carácter informativo o instructivo, de identificar información específica e incluso de inferir algún dato o intención que no estén presente en el enunciado.

- Prueba de comprensión audiovisual: consta de 3 tareas y una duración de 25 minutos. Su objetivo es mostrar al aprendiz una situación comunicativa propia del ámbito administrativo y laboral (en diferentes vídeos) y plantearle preguntas que requieran que active los mismos procesos y estrategias de comprensión que activaría en una situación similar en su contexto real. La prueba debe comprobar que el candidato puede comprender la idea principal de una conversación breve contextualizada; puede extraer datos concretos y puede inferir datos que no están presentes en el enunciado, a partir de recursos lingüísticos y no lingüísticos, acerca de la relación entre los interlocutores y la situación comunicativa.

- Prueba de expresión e interacción escrita: consta de 3 tareas y una duración de 20 minutos. Requiere la medición del desempeño de las destrezas de comprensión lectora y de expresión de manera integrada. Se trata de un tipo de evaluación subjetiva, por lo que los textos escritos por el candidato deben ser evaluados por dos evaluadores expertos de manera que se garantice la fiabilidad de la prueba. Para ello se realiza una evaluación holística, centrada en la competencia comunicativa funcional en lengua española y una evaluación analítica que tiene en cuenta los siguientes aspectos de la expresión e interacción escritas: expresión e interacción global; organización, cohesión y coherencia discursiva; competencia sociopragmática; alcance y control de vocabulario; alcance y control gramatical; y control ortográfico. Se evalúan las microhabilidades que constituyen la competencia de expresión e interacción escritas, verificando que el candidato se expresa, ofreciendo la información solicitada, cumpliendo los fines funcionales, manifestando adecuación al género textual, utilizando frases y oraciones breves y sencillas, enlazadas con conectores muy básicos.

- Prueba de interacción y expresión oral: consta de una entrevista en la que el candidato interviene en 3 conversaciones, con una duración de 10 minutos. Intervienen un examinador que actúa como interlocutor del candidato y un evaluador/ observador que presencia el examen, pero no puede intervenir en él, sino que se limita a valorar la actuación del candidato. Para realizar la evaluación con 
referencia a un criterio, en el caso de la evaluación analítica, hay diez categorías en el eje horizontal -mantenimiento de la conversación, cumplimiento de la intención comunicativa, fluidez, organización y cohesión/coherencia discursiva, competencia sociopragmática, alcance y control de vocabulario, alcance y control gramatical, control fonológico/ pronunciación, control fonológico/ entonación, y referentes socioculturales- mientras que el eje vertical lo constituye una escala de 1 a 4, en la que 1 indica el nivel más bajo de dominio lingüístico. Por otra parte, para demostrar estas capacidades se requiere que el candidato demuestre las siguientes microhabilidades lingüísticas: puede utilizar enunciados breves, sencillos, aislados; puede pronunciar y entonar con la adecuación suficiente para no impedir la comprensión; puede emplear un repertorio léxico y gramatical básico suficiente para alcanzar la intención comunicativa y puede utilizar fórmulas de cortesía sencillas y cotidianas para establecer contactos sociales básicos; puede organizar el discurso con coherencia y cohesión.

c) Validación de la prueba piloto. Para poder analizar la fiabilidad y validez de la prueba se aplicó esta a 184 informantes voluntarios de 18 a 58 años, de 30 nacionalidades y 50 lenguas maternas, en 10 centros (ONGs, CEPIs, y asociaciones). Según Nunnally (1994) los procedimientos estadísticos utilizados para este análisis deben aportar información sobre: la dificultad del instrumento (media de los índices de facilidad de los ítems que componen); el poder de la discriminación de la prueba (correlaciones biseriales puntuales de los diferentes ítems); la capacidad de la prueba para medir con consistencia la comprensión verbal/audiovisual (índice de fiabilidad) y, por último, establecer intervalos de confianza en torno a las puntuaciones observadas para así estimar las puntuaciones verdaderas (error típico de medida de la prueba). Los resultados mostraron que la prueba de comprensión lectora podía considerarse una prueba fácil, bastante fiable $(0,80)$ y con una validez media $(0,52)$. La prueba de comprensión audiovisual también se consideró de escasa dificultad, muy fiable $(0,90)$ y con una validez media $(0,6)$.

Tabla 1. Resultados del análisis de fiabilidad y validez en comprensión lectora y audiovisual

\begin{tabular}{|l|c|c|}
\hline & Comprensión lectora & Comprensión audiovisual \\
\hline Número ítems & 11 & 19 \\
\hline Número medio de aciertos & 8,79 & 13,8 \\
\hline Facilidad media & 0,80 & 0,73 \\
\hline Rbp media & 0,52 & 0,6 \\
\hline Fiabilidad ( Alfa de Cronbach) & 0,80 & 0,90 \\
\hline Error típico de la medida & 1,1 & 1,5 \\
\hline
\end{tabular}

Para analizar las pruebas holísticas y analíticas se realizó el cálculo de la correlación intraclase que mide la conformidad o consistencia de una serie de datos recogidos por varios evaluadores. Las correlaciones obtenidas fueron muy altas mostrando la fiabilidad de estas evaluaciones $(\mathrm{r}=0,947, \mathrm{p}=0,00)$. 


\subsection{Fase de formación de evaluadores}

Se consideró necesario diseñar e impartir dos cursos de formación que facilitasen la implantación del examen, preparando a los profesores que enseñan la lengua española en los diferentes centros de acogida así como a los evaluadores del examen. El Curso de Formación Especifica para Profesores de Español a Inmigrantes Adultos tenía como propósito principal ofrecer a los participantes un panorama didáctico y pragmático sobre las clases de español como segunda lengua (EL2) para este colectivo. Se pretendía conseguir que los participantes dispusiesen de un panorama general y específico de la situación migratoria en España, y adquiriesen recursos metodológicos básicos para actuar en la enseñanza del español a grupos de inmigrantes. El curso atiende a las necesidades lingüísticas específicas que suelen tener las personas inmigrantes que se encuentran en inmersión en la comunidad de acogida. Es habitual que se desarrollen las habilidades interpretativas con rapidez mientras que necesitan una mayor práctica en el uso de la lengua (Baralo, 2009; Baralo y Guerra, 2010). De ahí que el curso sensibilice sobre la importancia de la modalidad pragmática frente a la modalidad gramatical en la enseñanza del uso del español. La metodología empleada se basa en la interacción del alumno con el profesor tutor, del alumno con sus compañeros de curso, y del alumno con el propio itinerario formativo que contiene diversas actividades de comprobación de conocimientos con retroalimentación y autoevaluación. El contenido gira en torno a las necesidades formativas de los participantes y parte de sus conocimientos previos. A través de la lectura de la información básica de las fichas de contenidos, y gracias a los múltiples espacios de reflexión individual y discusión grupal, se va construyendo el aprendizaje. Además el tutor, que adopta un rol de facilitador del aprendizaje, posibilita que los participantes puedan conectar los nuevos conocimientos, habilidades y actitudes adquiridas con la realidad del aula. La interacción entre este y los participantes es continua a través de las diferentes herramientas de la plataforma virtual. En todo momento los participantes en el curso han dispuesto del apoyo de un técnico informático, de la dirección académica, de un coordinador de cursos, y de la atención personalizada de un tutor en línea. El curso se realiza de forma asincrónica, aunque se acuerdan sesiones sincrónicas si es necesario.

La evaluación del dominio lingüístico y comunicativo es una tarea compleja, difícil de objetivar y al mismo tiempo, imprescindible. La evaluación de la expresión e interacción oral no puede realizarse a través de una prueba objetiva, por eso fue necesario realizar el Curso de Formación de Evaluadores y Certificadores.

Uno de los principales requisitos para la implantación de una prueba de interacción oral es la adecuada formación de los evaluadores que van a participar en el proceso, puesto que el papel y el comportamiento del interlocutor durante la realización de la prueba es una variable que puede afectar a la realización lingüística del candidato, y a la puntuación del examinador. Según Karavas y Delieza (2009), la inconsistencia y la variación son los principales factores que afectan a la validez y la fiabilidad de la prueba. Los autores recogen varios aspectos en los que se producen esta inconsistencia y variación: la laxitud o dureza a la hora de aplicar la escala de calificación, la forma de interpretar las escalas de calificación y los criterios de evaluación, la forma de adaptarse al nivel de dominio lingüístico de los candidatos, el apoyo proporcionado a los candidatos para facilitar la comprensión y la producción, y en el modo de provocar [elicitation] la realización comunicativa de los candidatos. Todas estas recomendaciones se tuvieron en cuenta en la formación de evaluadores. El curso de carácter semipresencial tuvo una carga lectiva de 4 créditos ECTS (100 horas de trabajo del alumno, 40 de las cuales están dedicadas a la prueba de interacción oral). 
Para mejorar la difusión del examen y de la formación se diseñó un Portal de Internet, a través del cual se describe, explica y ejemplifica el examen de certificación. Este portal de Internet de 'Español para Inmigrantes' constituyó tanto una herramienta de difusión del examen de certificación, como una herramienta al servicio de profesores y alumnos para el aprendizaje y enseñanza de L2, así como para la preparación del examen.

\subsection{Fase de certificación: resultados de la primera convocatoria}

En la primera convocatoria se inscribieron 295 candidatos y 281 candidatos se presentaron al examen. El 66\% de los trabajadores que se presentaron tenía entre 17 y 37 años de edad, disminuyendo el número de presentados según aumenta la edad. Respecto al sexo, la distribución fue proporcional (49\% mujeres, 51\% hombres). El 53\% de los participantes procedían del continente europeo y el $41 \%$ del continente africano. Los lugares de procedencia más frecuentes son Rumanía (53\%), Ucrania (46\%) y Marruecos (37\%). También llama la atención la escasa frecuencia de la población china (2\%) que se presentó al examen.

Para otorgar las calificaciones se aplicó una escala de 0 a 10 , por considerarla la más habitual en el sistema educativo español, y se acompañó la nota numérica de una evaluación cualitativa, de acuerdo a una escala de cuatro puntos: No apto $(0-4,99)$, Satisfactorio $(5-6,59)$, Bien $(6,6-7,99)$ y Muy bien (8-10). Se recogen en la tabla 2 los resultados del examen por pruebas y según la calificación final, agrupados de acuerdo con las franjas de evaluación cualitativa, e incluyendo la categoría de candidatos no presentados al examen (NP).

Tabla 2. Distribución de calificaciones obtenidas en la certificación

\begin{tabular}{|l|c|c|c|c|c|}
\hline & No apto & Satisfactorio & Bien & Muy bien & Np \\
\hline Comprensión lectora & $18 \%$ & $14,67 \%$ & $13,67 \%$ & $47,33 \%$ & $6 \%$ \\
\hline Comprensión audiovisual & $5,33 \%$ & $7,33 \%$ & $8,33 \%$ & $72,67 \%$ & $6 \%$ \\
\hline Interacción escrita & $17,76 \%$ & $13 \%$ & $21,33 \%$ & $41,67 \%$ & $6 \%$ \\
\hline Interacción oral & $5,67 \%$ & $18,33 \%$ & $11,67 \%$ & $58,99 \%$ & $6 \%$ \\
\hline Notal final & $8,33 \%$ & $13,33 \%$ & $17,50 \%$ & $54,33 \%$ & $6 \%$ \\
\hline
\end{tabular}

Esta primera convocatoria gozó de una alta participación poco frecuente en este tipo de pruebas (297 participantes). El examen fue superado por el $92 \%$ de los presentados y con altas calificaciones (un 54\% de los candidatos presentados al examen obtuvo la máxima calificación).

Las pruebas de comprensión audiovisual e interacción oral registraron un porcentaje mucho menor de suspensos que las pruebas de destrezas escritas. Este último factor ya se tuvo en cuenta en la ponderación de la calificación de cada prueba sobre la nota final por lo que no requerirá ninguna otra consideración especial en próximas convocatorias. Sí se realizarán adaptaciones para ajustar los niveles de dominio lingüístico y la dificultad de la prueba.

Un amplio porcentaje de los candidatos (más del 80\%) logró un nivel de dominio lingüístico superior al A2 que medía el diploma (esta información se obtuvo del análisis individual de cada examen). 


\subsection{Evaluación del proceso de certificación}

Para mejorar las futuras convocatorias se consideró necesario conocer el impacto que la participación en este proceso de certificación había tenido en los diferentes agentes implicados. Por ello se realizó una encuesta de satisfacción en la que participaron 14 centros colaboradores, 15 evaluadores y 68 candidatos a examen. En esta los encuestados valoraron los aspectos que se describen a continuación:

Tabla 3. Opinión de los centros y los evaluadores sobre el proceso de certificación

\begin{tabular}{|c|c|c|c|c|c|}
\hline \multicolumn{6}{|l|}{ Centros } \\
\hline & $1 *$ & 2 & 3 & 4 & 5 \\
\hline Información aportada por la universidad & $7 \%$ & & $13 \%$ & $53 \%$ & $27 \%$ \\
\hline Folletos informativos & & & $21 \%$ & $43 \%$ & $36 \%$ \\
\hline Web del examen & & & $23 \%$ & $31 \%$ & $46 \%$ \\
\hline Materiales para la inscripción & & $7 \%$ & $29 \%$ & $21 \%$ & $43 \%$ \\
\hline Necesidad de material adicional & $7 \%$ & & $7 \%$ & $14 \%$ & $71 \%$ \\
\hline Depósito de inscripciones & $29 \%$ & & $14 \%$ & $29 \%$ & $29 \%$ \\
\hline Entrega de resultados & & $7 \%$ & $21 \%$ & $7 \%$ & $64 \%$ \\
\hline Web de profesores & & & $33 \%$ & $25 \%$ & $42 \%$ \\
\hline Guía didáctica & & & $25 \%$ & $50 \%$ & $25 \%$ \\
\hline \multicolumn{6}{|l|}{ Evaluadores } \\
\hline & $1 *$ & 2 & 3 & 4 & 5 \\
\hline Contenidos del curso on line & & & $8 \%$ & $46 \%$ & $46 \%$ \\
\hline Herramienta del curso on line & & & $8 \%$ & $46 \%$ & $46 \%$ \\
\hline Organización & & & & $15 \%$ & $85 \%$ \\
\hline Sesión presencial inicial & & & & & $100 \%$ \\
\hline Formación para la prueba oral & & & $15 \%$ & $23 \%$ & $62 \%$ \\
\hline Página web & & & $15 \%$ & $23 \%$ & $62 \%$ \\
\hline Información de la web & & & $0 \%$ & $46 \%$ & $54 \%$ \\
\hline Ejemplos de examen & $8 \%$ & & $46 \%$ & $15 \%$ & $31 \%$ \\
\hline Significatividad de las tareas del examen & $8 \%$ & & $46 \%$ & $15 \%$ & $31 \%$ \\
\hline Material gráfico de las pruebas escritas & $8 \%$ & $8 \%$ & $62 \%$ & $23 \%$ & \\
\hline Vídeos & & $8 \%$ & $46 \%$ & $46 \%$ & \\
\hline Material gráfico de la prueba de expresión e interacción oral & & $8 \%$ & $23 \%$ & $69 \%$ & \\
\hline Organización de la prueba oral & & $8 \%$ & $38 \%$ & $54 \%$ & \\
\hline
\end{tabular}

*1. Poco Adecuado - 5. Muy adecuado 
Si bien la valoración general del examen fue muy positiva los centros creen que deben ampliarse los materiales que permiten preparar la convocatoria, así como mejorar el sistema de depósito y de difusión de resultados. Se aprecia una aceptación generalizada de las vías de difusión (folletos y web), así como de la guía didáctica para preparar a los candidatos y del sistema de notificación de resultados. La mayoría de los centros $(86 \%)$ acogería un nuevo curso específico para la preparación del examen. Respecto a otras sugerencias de mejora los centros inciden en la necesidad de contar con más tiempo para realizar la difusión y sobre todo la preparación de los candidatos.

El Curso de Formación también fue bien valorado por los evaluadores. No obstante se realizaron interesantes sugerencias para mejorar los ejemplos, tareas, actividades didácticas, modelos de exámenes y el material gráfico (en especial el de la prueba de interacción oral).

En cuanto al nivel de dominio lingüístico que medía el examen, el $86 \%$ de los evaluadores consultados consideraron que era equiparable a un nivel A2.

Respecto a las características del examen, 68 candidatos opinaron sobre el nivel de adecuación de la web de preparación para el examen (http://www.diplomaletra.com), el nivel de dificultad de la prueba, y los tiempos asignados para realizar el examen. Respecto a la realización del examen, los candidatos se muestran conformes de manera bastante generalizada respecto a la dificultad (51\%) y al tiempo (90\%) dedicado a cada prueba. Cotejando estas opiniones con las de los evaluadores, podríamos considerar un reajuste del tiempo, para disminuir la duración de la prueba de comprensión audiovisual e incrementar la de la prueba de interacción escrita.

\section{Conclusiones}

El impacto obtenido por el diseño y la implantación de la Certificación lingüística comunicativa inicial en lengua española, para la obtención del Diploma LETRA, ha sido positivo y fructífero desde una perspectiva académica, intercultural, social e investigadora. Se ha cumplido con los estándares de fiabilidad y validez; se han introducido modelos de enseñanza/aprendizaje comunicativos que pueden servir de apoyo eficaz a los centros de español para inmigrantes; y se ha incluido la evaluación certificativa como meta del proceso de aprendizaje de la lengua con una metodología innovadora basada en el enfoque por tareas, que optimiza las posibilidades de interacción con los hablantes nativos, así como de integración en la sociedad de acogida. En cuanto al impacto intercultural, el diseño de la prueba ha permitido incluir todos aquellos contenidos que los estudios del Consejo de Europa han demostrado que facilitan las relaciones entre el nativo y "el otro", pues el examen LETRA ha incluido el acercamiento gradual a la cultura, a los referentes culturales, a los conocimientos socioculturales institucionales, y a los referentes cotidianos (horarios, costumbres laborales y empresariales, cortesía jerárquica y entre compañeros, entre otros muchos). La lengua es el vehículo de transmisión de los estos valores culturales, por lo que un dominio mayor de la competencia comunicativa en español garantiza contar con el instrumento esencial para las interacciones interculturales. En cuanto a la transferencia de la investigación realizada, se ha conseguido direccionarla en dos sentidos complementarios. Por un lado, a la comunidad investigadora en innovación educativa para la enseñanza de lenguas extranjeras, ya que se ha aplicado el concepto de evaluación como parte integrada y motivadora del proceso de 
aprendizaje de la lengua nueva. Por otro, la transferencia de resultados ha llegado aplicada a la didáctica y accesible para todos los profesores de español para inmigrantes que, de manera profesional o voluntaria, trabajan en los centros que acogen a los inmigrantes y les ofrecen una variedad de cursos de lengua y cultura española. La página dedicada a los profesores y la guía didáctica ofrece toda la información necesaria para conocer en profundidad cada una de las tareas comunicativas del examen, el diseño curricular, las sugerencias metodológicas y el material de apoyo para las clases. Permitiendo a estos profesionales mejorar su labor docente, usando los géneros discursivos y tareas que incluye este innovador diseño de examen de certificación lingüística comunicativa.

\section{Bibliografía}

Alderson, J., Clapham C. \& Wall, D. (1998). Exámenes de idiomas. Elaboración y evaluación. Madrid: Cambridge University Press.

Bachman, F. (1990). Fundamental considerations in language testing. Oxford: Oxford University Press.

Baralo, M. (2009). "Implantación de una certificación de nivel A1+1 y diseño curricular específico de español para trabajadores inmigrantes", en Actas del XX Congreso Internacional de ASELE. Santander: Fundación Comillas, 39-49.

Baralo, M. y Estaire, S. (2010). "Tendencias metodológicas postcomunicativas", en Abelló Contesse, C., Ehlers, C. y Quintana Hernández, L. (eds.), Escenarios bilingües: el contacto de lenguas en el individuo y la sociedad. Berlín: Peter Lang, 105-128.

Baralo, M. y Guerra, M.R. (2010). "Criterios pragmáticos y socioculturales para la selección de textos y géneros discursivos en un examen de certificación de nivel inicial (A2-n) de legua española para trabajadores inmigrantes". En Actas del XXI Congreso Internacional de ASELE. Salamanca: Universidad de Salamanca, 201-211.

Bordón, T. (2006). La evaluación de la lengua en el marco de E/L2: Bases y procedimientos. Madrid: Arcolibros.

Consejo de Europa (2002). Marco común europeo de referencia para las lenguas: aprendizaje, enseñanza, evaluación. Madrid: Ministerio de Educación, Cultura y Deporte, y Anaya.

Escudero, I. y Guerra, R. (2009). "La observación etnográfica como método para el análisis de necesidades comunicativas de la población inmigrante", en Actas del VII Congreso Internacional de AESLA .Ciudad Real: Universidad de Castilla la Mancha, 432-440.

Estaire, S. (2009). El aprendizaje mediante tareas: de la programación al aula. Madrid: Edinumen.

Fernández, S. (2003). Propuesta curricular y Marco común europeo de referencia. Desarrollo por tareas. Madrid: Edinumen / Ministerio de Educación, Cultura y Deportes.

García Mateos C. (2004). Experiencias y propuestas para la enseñanza de L2 a personas inmigradas. Madrid: Edinumen.

Herrero, M. (2013). "Análisis de la utilización de estrategias en el aprendizaje de la lengua extranjera", en Revista de Investigación educativa, 31, 1:53-76.

Instituto Cervantes (2006). Español como nueva lengua. Orientaciones del Instituto Cervantes para un curso de emergencia destinado a inmigrantes. Madrid:Santillana.

Karavas, E. \&Delieza, X. (2009).“'On site observation of KPG oral examiners: Implications for oral examiner training and evaluation", en Apples-Journal of Applied Language Studies, 3,1:51-77. 
Luoma, S. (2004). Assessing speaking. Cambridge: Cambridge University Press.

Llorián González, S. (2012). "Los retos de la certificación del español con fines específicos", en Revista Nebrija de Lingüistica Aplicada 12 ,6:3-34.

Martín Leralta, S. (2009). "Español con fines profesionales: necesidades de comprensión de los inmigrantes en los ámbitos administrativo y profesional", en Actas del XX Congreso Internacional de ASELE. Santander: Fundación Comilla, 719-738.

Martín Leralta, S. (2011). "La evaluación de la interacción oral para la certificación lingüística en L2", en XVI Congreso Internacional de la ALFAL. Alcalá de Henares:Universidad de Alcala, 122-125.

Martínez Clares, P. Echeverría B. (2009). "Formación basada en competencias", en Revista de Investigación Educativa, 27, 1: 125-147.

Nunnally J. C. (1994). Psychometric theory. New York: McGraw Hill

Aguado-Orea, J. y Baralo, M. (2007). "Aspectos teóricos y metodológicos de la investigación sobre el aprendizaje léxico y gramatical del español como L2" en Revista de Educación, 343:113-132.

Vilà, R. (2006). "La dimensión afectiva de la competencia Comunicativa intercultural en la Educación secundaria obligatoria: Escala de sensibilidad intercultural", en Revista de Investigación Educativa, 24, 2: 353-372.

Villalba, F. y Hernández, T. (2007). "La enseñanza de español con fines laborales para inmigrantes" .Linred Lingüistica en la Red, 5. Disponible en: http://www.linred.es/numero5_anexo1_Art3.html, consultado el 20 de septiembre, 2012. 